\title{
Interdisciplinary endoscopic assisted surgery of a patient with a complete transorbital intracranial impalement through the dominant hemisphere
}

\author{
Burkhardt, J K ; Holzmann, D ; Strobl, L ; Woernle, C M ; Bosch, M M ; Kollias, S S ; Reisch, R
}

DOI: https://doi.org/10.1007/s00381-011-1674-8

Posted at the Zurich Open Repository and Archive, University of Zurich ZORA URL: https://doi.org/10.5167/uzh-64753

Journal Article

Accepted Version

Originally published at:

Burkhardt, J K; Holzmann, D; Strobl, L; Woernle, C M; Bosch, M M; Kollias, S S; Reisch, R (2012). Interdisciplinary endoscopic assisted surgery of a patient with a complete transorbital intracranial impalement through the dominant hemisphere. Child's Nervous System, 28(6):951-954.

DOI: https://doi.org/10.1007/s00381-011-1674-8 


\section{Interdisciplinary Endoscopic Assisted Surgery of a Patient with a Complete}

\section{Transorbital Intracranial Impalement through the Dominant Hemisphere}

Jan-Karl Burkhardt, M.D. ${ }^{1,2}$, David Holzmann, M.D. ${ }^{3}$, Lisa Strobl, M.D. ${ }^{1}$, Christoph M. Woernle, M.D. ${ }^{1}$, Martina M. Bosch, M.D. ${ }^{4}$, Spyros S. Kollias, M.D. ${ }^{5}$ and Robert Reisch, M.D. ${ }^{1,6,+}$

${ }^{1}$ Department of Neurosurgery, University Hospital, University of Zurich, Switzerland

${ }^{2}$ Department of Neurological Surgery, Weill Cornell Medical College, New York Presbyterian Hospital, New York, NY, USA

${ }^{3}$ Department of Otorhinolaryngology, Head and Neck Surgery University Hospital, University of Zurich, Switzerland

${ }^{4}$ Department of Opthalmology, University Hospital, University of Zurich, Switzerland

${ }^{5}$ Department of Neuroradiology, University Hospital, University of Zurich, Switzerland

${ }^{6}$ Center for Endoscopic and Minimally Invasive Neurosurgery, Clinic Hirslanden, Zurich, Switzerland

+ Corresponding author

Address for correspondence:

Robert Reisch, $M D$

Centre for Endoscopic and Minimally Invasive Neurosurgery,

Clinic Hirslanden

Witellikerstrasse 40

8032 Zürich, Switzerland

phone: $+41 / 443872853$

fax : $+41 / 443872861$

email: robert.reisch@hirslanden.ch 


\begin{abstract}
Intracranial impalement injuries through a transorbital entry route are rarely reported in literature. Here, we describe a case of a 4 year old boy presenting with a complete transcranial penetration of a thick wooden stick through the dominant hemisphere, operated successfully with a combined transnasal-transcranial approach. The penetrating channel ranged from the left inner orbital angle to the right occipital bone by fracturing the lamina papyracea, the lateral wall of the sphenoid sinus and penetrating the cavernous sinus as well as the dominant temporo-parieto-occipital brain tissue. Interdisciplinary rhinosurgical-neurosurgical endoscopic assisted surgery was performed and the wooden foreign body was removed completely. After surgery, a traumatic carotid-cavernous fistula was diagnosed and occluded with interventional endovascular technique. The hemiparesis, aphasia and right ophthalmoplegia showed complete recovery; amaurosis of the right eye remained unchanged. The ipsilateral eye, which had been shifted laterally, showed no deficits. In this case the combined transnasal-transcranial approach allowed safe and successful removal of the foreign object and the boy returned to everyday life with a favourable neurological outcome after one year follow-up.
\end{abstract}

Keywords Endoscopic assisted surgery, transorbital intracranial impalement, combined transnasal-transcranial surgery, interdisciplinary approach 


\section{Introduction}

Transorbital injuries are common and represent about $24 \%$ to $45 \%$ of all penetrating head injuries in adults and children.[8] However, only $<1 \%$ of the cases show a skull or brain penetrating route with consecutive intracranial injuries.[2, 8] The direction and orbital entry route depends on the velocity of the impalement and the trajectory of the foreign body. In low-velocity injuries the foreign body may be directed by the configuration of the orbital walls and enters most likely through the superior and inferior orbital fissures or the orbital canal.[1] In cases of higher velocity impalements the orbital wall may directly fracture following a perpendicular trajectory line. Here we describe the first case in the literature of a complete impalement injury through the dominant hemisphere entering the inner orbital angle, fracturing the medial aspects of the skull base and leaving the skull contralaterally through the occipital bone, treated successfully in interdisciplinary cooperation via a combined transnasal - transcranial approach.

\section{Case Report}

\section{Patient history and clinical examination}

A 4-year-old left handed boy was transferred to our department after a traumatic impalement injury through his left eye while playing with building bricks at home. Initially, he presented with a rapid progressive loss of consciousness at the University Childrens Hospital Zurich with a wooden stick $(30 \mathrm{~cm} \times 1 \mathrm{~cm})$ from a building set entering the medial angle of his left eye displacing the left globe laterally and inferiorly (Fig. 1 A-C). The assessment of the left pupil was difficult due to the surrounding swelling, but showed normal afferent and efferent function of the pupil. The right eye showed a dilated pupil without reaction to light. The foreign body penetrated through the left superior eye lid. 
CT scan of the head confirmed the impalement with a transcranial penetrating route through the contralateral side, fracturing the occipital bone (Figure 1). The penetrating route led from the nasal half of the left upper eyelid fracturing the lamina papyracea, the posterior ethmoidal cells to the contralateral sphenoid sinus on the right. Here the wooden foreign body continued penetrating the right cavernous sinus and was in close contact to the cavernous segment of the internal carotid artery. Further posterior, the middle cranial fossa was entered and left the right-sided occipital bone after piercing through the temporo-parieto-occipital brain tissue. No major intracranial bleeding was seen on the CT.

\section{Operative course}

After transferring the boy to the operating room, interdisciplinary endoscopic assisted emergency surgery (neurosurgery, ENT surgery and ophthalmology) was performed. Since we were uncertain whether the ICA was injured by the foreign body or could be injured by manipulating the latter, we decided to expose the contact area of the foreign body with the ICA from below and above to guarantee a secure exposure and to be able to manage potential bleeding out of the ICA. First a transnasal endoscopic approach was performed (D.H.) exposing the ICA in its proximal segment as well as the foreign body from below using a wide right sphenoethmoidectomy. To expose the cavernous segment of the ICA and the optic canal the small sphenoid sinus was widely opened and the foreign body was seen exactly in the right lateral opto-carotid recess (Figure 2). Thereafter, a pterional craniotomy was performed (R.R.) and the skull base was inspected through the transcranial way. The wooden stick was found entering the middle fossa through the lateral wall of the cavernous sinus. The stick was completely blocked within the skull base and could not be removed. As there was a high potential risk of rupturing adjacent vessels by rough removal of the stick we decided to cut it into two pieces above the level of the anterior knee of the ICA using a high-speed drill (Figure 2). The anterior piece was carefully removed first under continuous transnasal 
endoscopic and transcranial microscopic monitoring. The simultaneous visualisation allowed safe proximal and distal control of the carotid artery; there was no severe bleeding, however the right optic nerve showed a complete rupture. The right internal carotid artery (ICA) appeared spastic and only limited blood flow was detected by Doppler sonography. Therefore, after removal of the posterior part of the stick, the pterional craniotomy was enlarged for additional decompression prior to closure. The upper eyelid wound was sutured after inspection by the ophthalmic surgeon (M.M.B.). Postoperative CT-scan showed no severe brain injury. However, CT-Angiography revealed a slight displacement and stenosis of the ICA and supposed a traumatic fistula. A digital subtraction angiography verified a carotidcavernous fistula and an occlusion of the vessel was performed in light of sufficient collateral supply of the affected dominant hemisphere (S.S.K.). (Figure 3).

\section{Postoperative course}

After surgery the patient was stable and transferred to the pediatric ICU. Intracranial pressure monitoring showed normal values; after weaning and extubation the patient showed a high grade left sided hemiparesis and global aphasia due to the injured dominant right hemisphere. The ophthalmic examination revealed ophthalmoplegia with mydriasis and amaurosis on the right side, whereas the left eye showed a normal reactive pupil, adequate motility and an unremarkable fundus. Three months after surgery, the frontoparietal bone was re-implanted with an uneventful postoperative course. At one year follow up and after neuro-rehabilitation the boy showed a nearly complete regression of his hemiparesis with a slight remnant of his motor dysfunction and no impairment in understanding and speech production. The rightsided ophthalmoplegia and mydriasis recovered completely, however, the right amaurosis remained unchanged (Figure 4). 


\section{Discussion}

In this report we present a case of a complete transcranial impalement through the patient's dominant hemisphere. Sharp or blunt transorbital impalement injuries are rarely described in children and occur most of the time during playing, as in this case.[2, 4-6, 8] Due to the thin anatomy of the orbita, where the bone is of least resistance, high-velocity injuries may lead to a continuation of the impalement intracranially.

In our case the penetrating channel of the wooden foreign body involving the cavernous sinus potentially indicated injury of the carotid artery, thus necessitating safe proximal and distal control of a possible intraoperative fatal bleeding; this could be achieved through the combined transnasal-transcranial approach by exposing the wooden foreign body from medial and below and from lateral and above, respectively. With this combined approach the ICA could be exactly displayed and a bleeding event would have been precisely controlled, which did not occur.

Endoscopic transnasal skull base surgery for young children is rarely described in the literature, especially if the cavernous sinus and the cavernous segment of the ICA need to be exposed.[3] We showed for the first time that our interdisciplinary transnasal-transcranial approach is a feasible and secure method to treat a traumatic impalement in a 4 year old child. Since age-related differences in the anatomy of the skull base may limit endoscopic transnasal surgery in children this technique should be performed by experienced endoscopic surgeons.[7]

Here, we describe to our knowledge the first case of a complete transcranial penetration of a wooden foreign body, treated successfully via a combined transnasal-transcranial approach with a favourable clinical patient outcome after one year. 


\section{References}

[1] Balasubramanian C, Kaliaperumal C, Jadun CK, Dias PS (2009) Transorbital intracranial penetrating injury-an anatomical classification. Surg Neurol 71: 238-240

[2] Dunn IF, Kim DH, Rubin PA, Blinder R, Gates J, Golby AJ (2009) Orbitocranial wooden foreign body: a pre-, intra-, and postoperative chronicle: case report. Neurosurgery 65: E383-384; discussion E384

[3] Kassam A, Thomas AJ, Snyderman C, Carrau R, Gardner P, Mintz A, Kanaan H, Horowitz M, Pollack IF (2007) Fully endoscopic expanded endonasal approach treating skull base lesions in pediatric patients. J Neurosurg 106: 75-86

[4] Lee JS, Lee JE, Oum BS, Cha SH (1999) Orbitocranial injury caused by wood. Korean J Ophthalmol 13: 128-132

[5] Orszagh M, Zentner J, Pollak S (2009) Transorbital intracranial impalement injuries by wooden foreign bodies: clinical, radiological and forensic aspects. Forensic Sci Int 193: $47-55$

[6] Park SH, Cho KH, Shin YS, Kim SH, Ahn YH, Cho KG, Yoon SH (2006) Penetrating craniofacial injuries in children with wooden and metal chopsticks. Pediatr Neurosurg 42: $138-146$

[7] Tatreau JR, Patel MR, Shah RN, McKinney KA, Zanation AM (2010) Anatomical limitations for endoscopic endonasal skull base surgery in pediatric patients. Laryngoscope 120 Suppl 4: S229

[8] Turbin RE, Maxwell DN, Langer PD, Frohman LP, Hubbi B, Wolansky L, Mori M (2006) Patterns of transorbital intracranial injury: a review and comparison of occult and non-occult cases. Surv Ophthalmol 51: 449-460 


\section{Figure Legend}

Figure 1: (A-B) Preoperative clinical images of the patient with the transorbital intracranial impalement. (C-G) Axial CT images show the penetrating route of the wooden foreign body (arrow). (H) CT 3D reconstruction of the skull shows the fractured occipital bone.

Figure 2: (A-B) Transnasal intraoperative endoscopic view: (A) the wooden foreign body (asterisk) was exposed next to the lateral wall of the right sphenoid sinus (SS) and the planum sphenoidale (PS). (B) After removal of the wooden foreign body the ICA was intact without signs of injury. (C-D) Transcranial intraoperative microscopic view before (C), during (D) and after (E) high-speed drilling of the wooden foreign body (asterisk) in two parts.

Figure 3: (A) Postoperative DS-angiography in sagittal plane verified a carotid-cavernous fistula. (B) Occlusion of the right internal carotid artery was performed after sufficient collateral supply of the right dominant hemisphere through the left arterial territory was confirmed.

Figure 4: (A-B) Postoperative clinical images of the patient show the favorable patient outcome with a regression of the left-sided hemiparesis and the right-sided ophthalmoplegia. 
Figure 1:
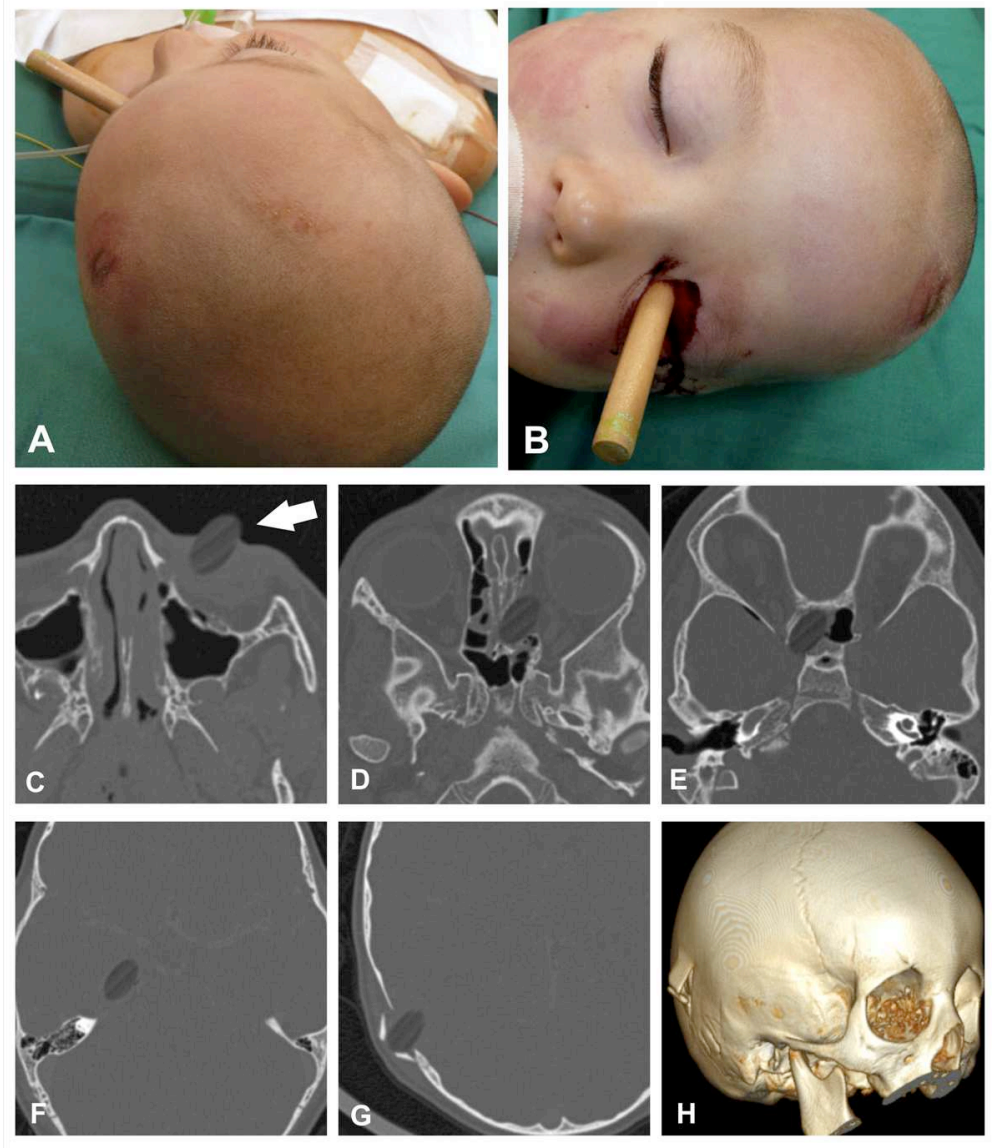

Figure 2:

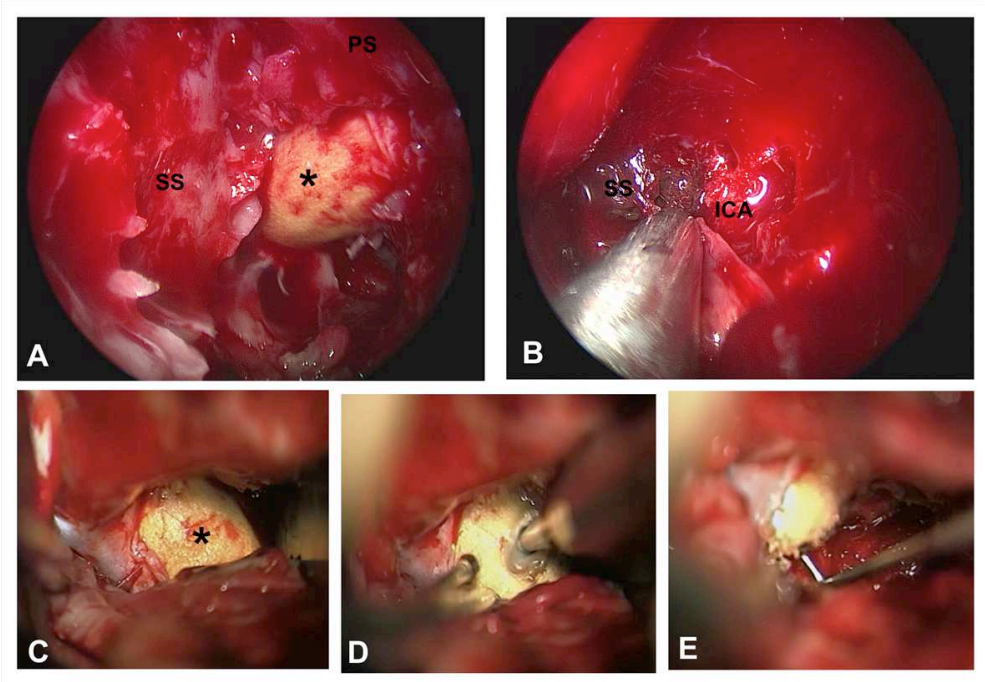


Figure 3:

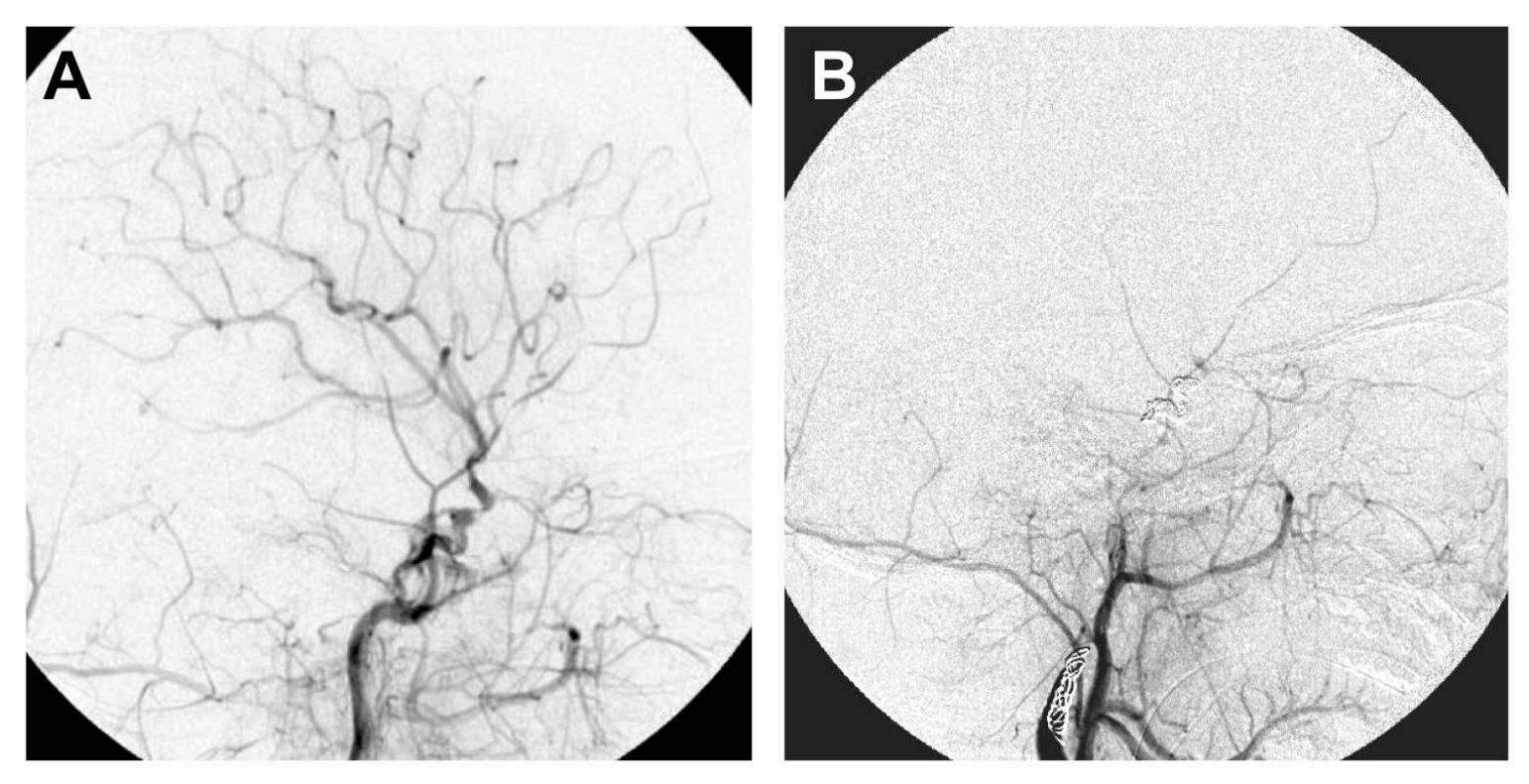

Figure 4:
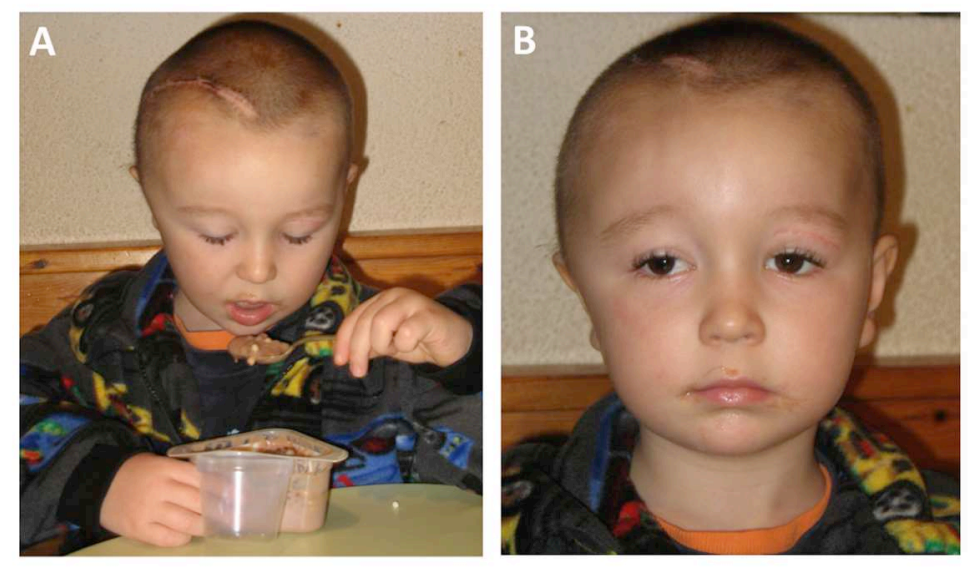